\title{
STUDY OF ADVANCES AND CHALLENGES IN GEOVISUAL
}

\section{ANALYTICS}

\author{
Mragank Singhal ${ }^{1}$ \\ ${ }^{1}$ College of Computing Sciences and Information Technology, Teerthanker Mahaveer University, Moradabad
}

\begin{abstract}
Geovisual Analytics is an emerging interdisciplinary field that integrates perspectives from Visual Analytics, GIS based services, particularly, on work of geovisualization, geospatial semantics and knowledge management paradigm This paper elaborates the role of this emerging field as well as also focuses over the applications of Geovisual Analytics.
\end{abstract}

This paper focuses over the emerging ternds and challenges coming in the vision of geovisual analytics. This paper emphasizes the importance of visualization and interactive visual interfaces and the link with the emerging research discipline of Visual Analytics

Keywords - GIS, Geovisual Analytics, Visual Analytics, geospatial, knowledge management

\section{INTRODUCTION}

Geospatial Analytics is a new paradigm for how information technologies can be used to process complex geospatial information to facilitate decision making, problem solving [3]. Nowadays, there are a lot of Geovisual tools that help to identify geospatial information. The end goal of Geospatial Analytics is the dissemination of results to decision makers as well as succinct communication of the interpretation made by an analyst or group of analysts [5].

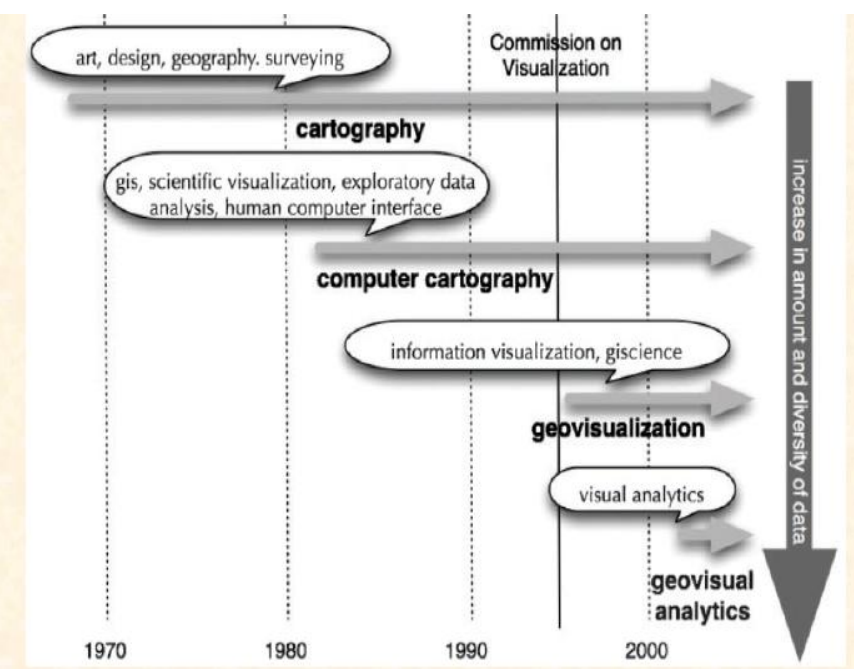

Fig 1

\section{VISUAL ANALYTICS AND RELATED}

\section{SCIENCES}

The background of Geospatial Analytics comes into existence with the concept of visual analytics. Visual analytics is the science of analytical reasoning facilitated by interactive visual interfaces [2].
A visual analysis process normally includes the following four phases:

1) processing and representing data

2) visualizing data

3) analyzing data and

4) Presenting and communicating analytical results.

Therefore, visual analytics includes the following four focus areas:

_ data representations and transformations that convert data in ways that support visualization and analysis

_ visual representations and interaction techniques which allow the user to see, explore and understand large amounts of data at once;

_ analytical reasoning techniques that enable users to gain deep insights that directly support assessment, planning and decision making.

_ techniques to support production, presentation and dissemination of the results of the analysis process to communicate information in the appropriate context to various types of audiences.

The major applications underlying are as follows:

- Analytical reasoning techniques that enable users to obtain deep insights that directly support assessment, planning, and decision making

- Visual representations and interaction techniques that take advantage of the human eye's broad bandwidth pathway into the mind to allow users to see, explore, and understand large amounts of information at once

- Data representations and transformations that convert all types of conflicting and dynamic data in ways that support visualization and analysis

- Techniques to support production, presentation, and dissemination of the results of an analysis to 
communicate info in the appropriate context to varied audiences

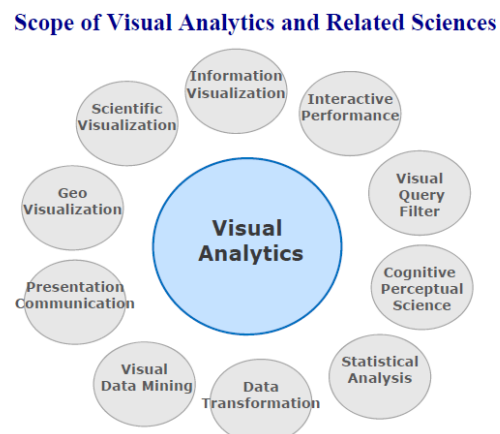

Fig 2

\section{FRAMEWORK}

The Geovisual analytics is an emerging interdisciplinary field which is based on the principles of visual analytics [1]. It can be considered as a sub-field of visual analytics but focuses on geographic aspects such as geospatial data, map techniques and geographic phenomena [7].

Geovisual analytics which focuses on spatial decision support this focus area deals with spatial decision problems which normally (1) have complex nature of geographic and temporal spaces, (2) involve multiple actors with different roles and (3) involve tacit criteria and knowledge. It aims at supporting users to make effective decisions through the use of automated algorithms, interactive visualizations and human analysis. Another key topic of geovisual analytics is 'Space and Time' which focuses on both space and time aspects and addresses the temporal nature of geographic data [6], the characteristics of time as well as the structure of time.

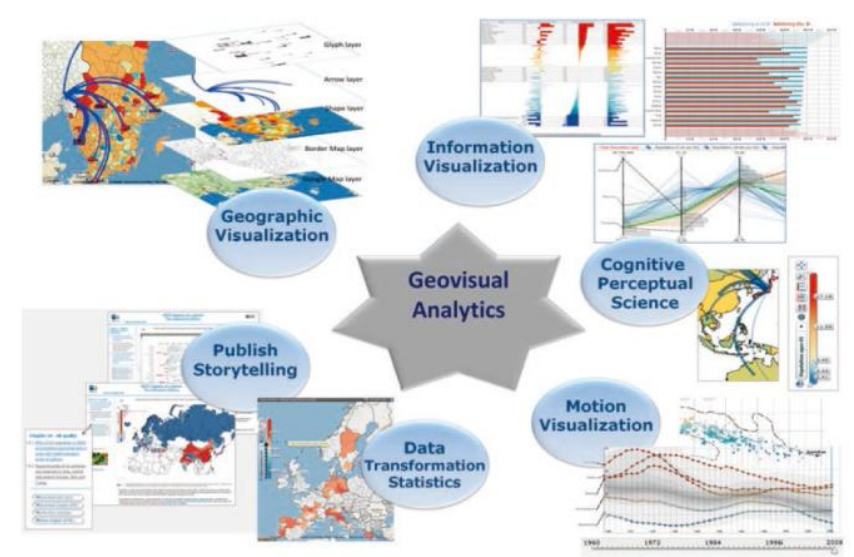

Fig 3 An overview of fields and techniques integrated into geovisual analytics.

To demonstrate the architecture of geovisual analytics ,a web based framework has been taken for setting an example.

The web-enabled framework is based on component thinking or the component based approach which is inherited from its predecessor. Based on this approach, the framework is built as a collection of components which allows application developers to pick and choose from a wide range of visualizations, data providers and data transforms and combine them in various ways they want. This facilitates the development of large-scale systems.

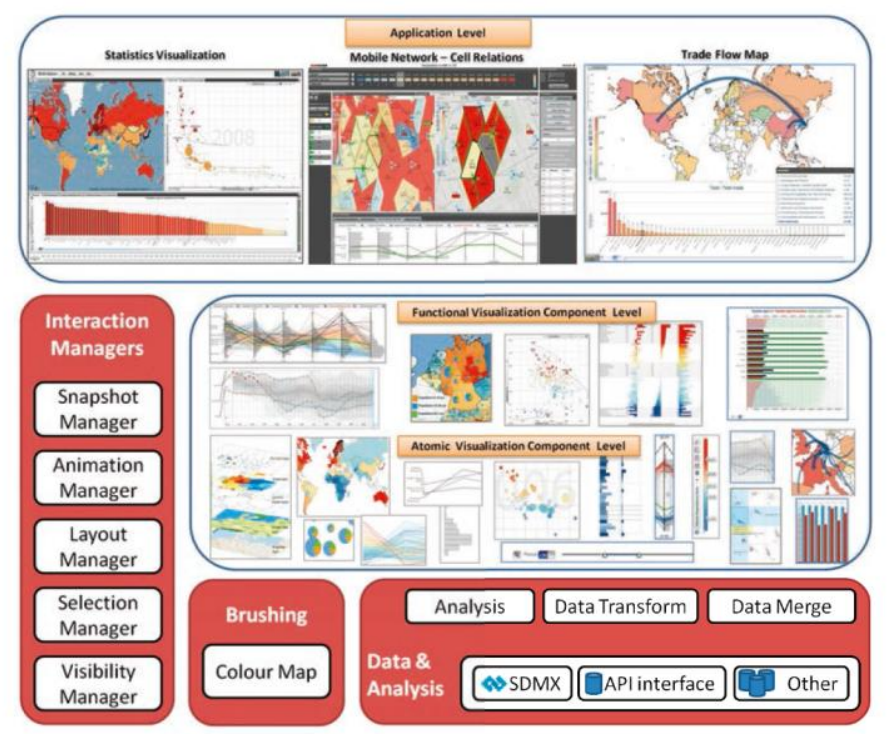

Fig 4 An overview of web based framework.

The framework is developed to include the following five component types:

- layout components which are for creating application layouts;

- data components which are for loading data, processing data and transforming data;

- visualization components which are for visualization of data and interaction with

- linking components which are for linking visualization components together to create multiplelinked views applications; and

- snapshot and storytelling components which are for capturing and saving the state of components and creating stories as well as publishing stories.

\section{APPLICATION AREAS OF GEOSPATIAL}

\section{ANALYTICS}

Geovisual Analytics has diversified its research areas [8].Some of them is demonstrated as:

- Visual Interfaces and Representations

- Collaboration/Group Work Support

- Analytical reasoning

- Problem solving and Decision Making

- Situation Awareness

- System Evaluation

Each of the above mentioned areas have been implemented into various practical aspect, some of them are discussed as follows:

\section{1) Crisis Management}

The major issues associated with crisis management are as follows

- Increasing diverse forms of geo-information 
- Huge volumes, Non-traditional sources

- Large and complicated analytical tasks

These issues are overcome using geovisual analytics technique, Consolidated Appeals Process (CAP), Context analysis is meant to "outline the root cause of the crisis and its evolution, including any regional or historical dimensions"

\section{2) The Context Discovery Application (CDA)}

- Geovisual Analytic tool facilitate visual interfaces to support analytical reasoning.

- Supports Asynchronous Geocollaboration of information related to the context.

- Enables Computational processing, geocoding and visualization of place names

- Provides the option of Automated reasoning for document ranking

3) Automated Reasoning and Document Ranking

This application is the most upcoming field where the usage of geovisual analysis is emerging rapidly. A Process flow diagram has been demonstrated below.

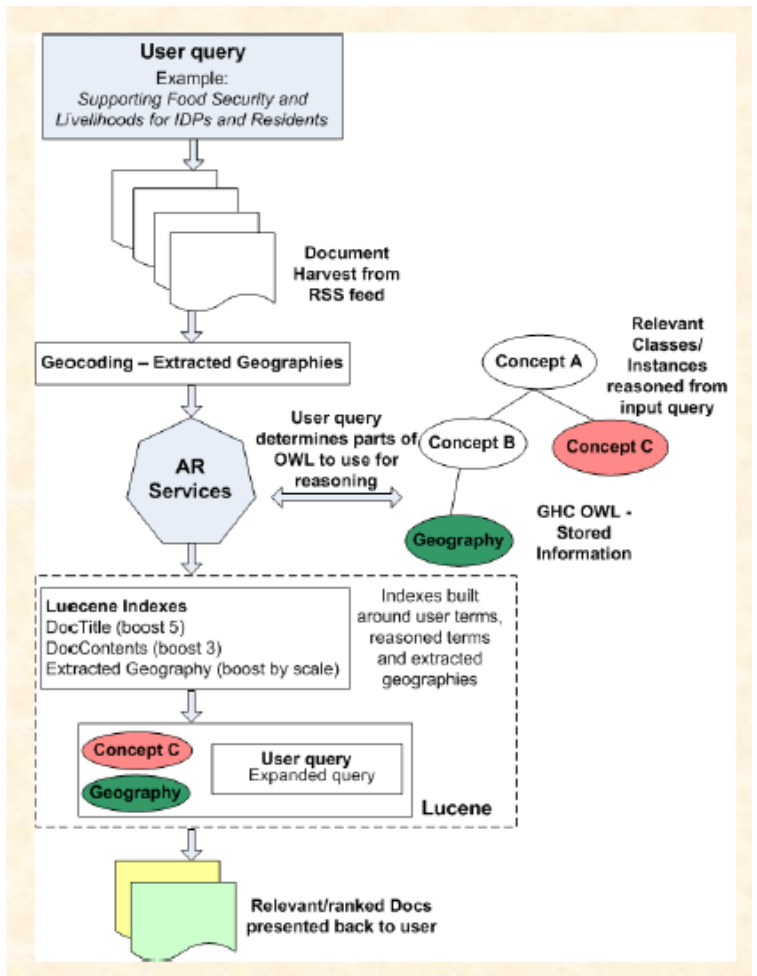

Fig 5: Process Flow block diagram of document ranking.

\section{CHALLENGES IN GEOVISUAL ANALYTICS}

Many advances have been made in the research of geovisual analytics but many challenges are still remaining.

Some of them have been discussed here:

1) The user wants to have more interaction techniques and interactive features in visualizations. For example, when working with scatter diagram, therefore requirement can be made for more interaction techniques and interactive features such as zoom, animation, colour changing, and so on. In general, it is still a challenge to combine a rich set of interaction techniques and interactive features into visualizations in a good way to support better analysis.

2) Possibility can be to capture discoveries made and save them so that user can share them to other users such as analysis consumers or the public. This feature requires effective methods for capturing and saving interactive visualizations so that other users can see and even interact with saved interactive visualizations to confirm the result or give feedback. In current practice, most of geovisual analytics systems lacking this feature.

3 ) In case of solving problems in different domains. There is still a big gap between the increasing needs of the user for analyzing data in many different domains and what analytics has contributed so far. The challenge comes from the fact that data in different geospatial domains normally have different characteristics and, in many cases, are very complex. For each specific type of data, techniques may vary. This challenge relates also to the fact that the research so far has not focused on the development of frameworks and systems which can reduce the time and effort for developing geovisual analytics applications.

4) Another challenge is to adapt geovisual analytics tools to the internet environment and mobile platforms so that they can reach a broader user community and the public can benefit from them.

5) The need for using geovisual analytics tools to disseminate data and analytical results in the form of interactive visualizations to get more attention from the public and the media on various issues is also increasing continuously; therefore, a common web based framework is required.

\section{CONCLUSIONS}

Most The researches in geovisual analytics is a new branch of visual analytics, with the vision of analytically implementing spatial data. Various advances have been made other yet to reach.

In summary it can be affirmed that:

1) This is a new framework which is web-enabled, extendible including a wide range of highly interactive visualizations which are enhanced with a rich set of interaction techniques and interactive features.

2) It supports different projections and online web map services;

3) Multiple coordinated and linked views through various linking mechanisms are provided.

4) Supports a library which simulates a multithreading environment to improve interactivity;

5) Facilitates the development of web-enabled interactive visualization applications.

6) Supports spatial and non spatial data formats which are widely used in the statistics community. 


\section{REFERENCES}

[1] Mehdi Amirijoo, P_al Frenger, Fredrik Gunnarsson, Harald Kallin, Johan Moe, and Kristina Zetterberg. Neighbor cell relation list and physical cell identity selforganization in 1te. In Proceedings of the 2008 IEEE International Conference on Communications, pages 37\{41, Washington, DC, USA, 2008. IEEE Computer Society.

[2] Richard A. Becker, Stephen G. Eick, and Allan R. Wilks. Visualizing network data. IEEE Transactions on Visualization and Computer Graphics, 1(1): $16\{28,1995$.

[3] Anselin, L. (2000): Computing environments for spatial data analysis, Journal of Geographical Systems, 2, 2000, pp. 201-220.

[4] Stuart K. Card, Jock D. Mackinlay, and Ben Shneiderman, editors. Readings inInformation Visualization: Using Vision to Think. Morgan Kaufmann, 1999.

[5] Malczewski, J. (1999): GIS and Multicriteria Decision Analysis, John Wiley \& Sons:New York, USA, 1999

[6] Malczewski, J. (2006): GIS-based Multicriteria Decision Analysis: a Survey of the Literature, International Journal of Geographical Information Science, 20 (7), August2006, pp. 703-726

[7] Nevo, D., and Wand, Y. (2005): Organizational memory information systems: a transactive memory approach. Decision Support Systems, 39(4), 549-562.

[8] Palmer, J. D., Fields, N. A., and Brouse, P. L. (1994): Multigroup Decision-Support Systems in CSCW, Computer, 27(5), pp. 67-72. 